Petr KReuZ, Prag

\title{
Die Gerichtsbarkeit der Prager Altstadt und der vereinten Prager Städte in der Zeit der Jagiellonen (1471-1526)
}

The judiciary of the Old Town of Prague and the United Prague towns in the time of the Jagiellons (1471-1526)

The purpose of this contribution is to present a research project on the judiciary of the Old Town of Prague and the United Prague towns in the time of the Jagiellons and some of its preliminary results. The main starting-point as to the investigated sources is represented by sentences and verdicts of the town court of the Old Town of Prague from 1475-1518 and by sentences and verdicts intimated by the town court of the United Prague towns from 1518-1526. The sentences and verdicts passed in those years are found predominantly in the Books of verdicts (libri sententiarum).

From the entire number of 1,004 registered sentences in law-suits, 533 were passed by the town court of the Old Town in 1475-1518, and 471 by the town court of the United Prague towns in 1518-1526. From those 1,004 sentences, 257 (25.6\%) concern criminal cases. Also in the paper, the results of the analysis of the abovementioned criminal cases are presented. The analysis led to the conclusion that the greater part of the criminal agenda of the courts under investigation consisted of criminal acts against life and health and honour. Offences against property occurred only in relatively smaller numbers.

Regarding the development of town law it is possible to register during the Jagellonian era a conspicuous improvement concerning the adjucative practice of the court of the Old Town. This improvement was reflected also in a better quality and more refined forms of decisions rendered by the court. Whereas the Old Town law is to be regarded from the point of view of its development as representing at the outset of the Jagellonian era in considerable measure an autochthonous legal system sui generis taking over only in an unsystematic way and ad hoc the norms of the south German and Magdeburg town laws, the first tenth of the epoch in question in the Old Town was characterised by the broad and intense taking over of the conspicuously more developed town law of Brno (Brünn) and of Roman law too. Towards the end of the Jagellonian era, this takeover in the Prague towns was completed not only at the theoretical level, but also in the sphere of legal practice, thereby creating one of the central preconditions for the later codification of the Bohemian town law (1579).

Keywords: criminal cases - Jagellonian era - Old Tow of Prague - Prague - sentences- town court - town law

Ziel des vorliegenden Beitrags ist eine kurze Darstellung des Projekts der komplexen Erforschung des Gerichtswesens der Prager Altstadt und der vereinigten Prager Städte in der Zeit der Jagiellonen und der ausgewählten bisherigen (und vorläufigen) Resultate dieser Erforschung.
Den Ausgangspunkt meiner Erforschung bilden die Urteile, Befunde und Schlichtungs-(Arbitrage-)Entscheidungen des Stadtgerichts der Prager Altstadt aus den Jahren 1475-1518 und diejenigen des Gerichts der vereinigten Prager Städte aus den Jahren 1518-1526. 
Die Altstadt Prag war die älteste der vier Städte, die das spätmittelalterliche und frühneuzeitliche Prag bildeten (die Prager Altstadt und die Prager Neustadt am rechten Moldauufer, die Kleinseite/Prager Kleinstadt und der Hradschin am linken Moldaufer gelegen). ${ }^{1}$ Gleichzeitig stellte die Altstadt Prag die volksreichste Stadt im Königreich Böhmen und in den Ländern der Böhmischen Krone dar; ihre Einwohnerzahl betrug in der jagiellonischen Zeit etwas mehr als 10.000 Einwohner. Im ausgehenden Mittelalter war die Prager Altstadt die politisch bedeutsamste Stadt innerhalb der Länder der Böhmischen Krone und (zusammen mit Leitmeritz) eines der Zentren des Stadtrechts und der Stadtgerichtsbarkeit im Königreich Böhmen. Im Jahre 1518 vereinte sie sich vorübergehend mit Prager Neustadt. Dadurch wurden die größten Prager königlichen Städte, beide am rechtem Moldauufer, vereinigt und die sogenannten vereinigten Prager Städte (verbundenes Prag) gebildet, die fast 20.000 Einwohner zählten. Die Einheit der Prager Städte wurde im Jahr 1528 von König Ferdinand I. aufgehoben. ${ }^{2}$

Aus der von den in Prag Altstadt sowie in den vereinten Prager Städten in der Zeit der Jagiellonen ausgeübten gerichtlichen Tätigkeit haben sich in fast ununterbrochener Reihe mehr als 1.000 Urteile und Gerichtsbefunde erhalten. Der gleichen Zeit entstammen etwa zwei Dutzend Sprüche in den Arbitrage- und Schlichtungsverfahren. ${ }^{3}$

Die obengenannten Befunde, Gerichtsentscheidungen und -sprüche stellen einen bedeutsamen Komplex von Quellen zur Rechts-, Wirtschaftsund Sozialgeschichte Prags im ausgehenden Mittelalter dar. Die vor dem Stadtgericht zur Verhandlung gelangenden Fälle bieten manchmal einen einmaligen Einblick in verschiedene Lebenssphären praktisch aller Einwohner-

\footnotetext{
${ }^{1}$ Zur Prager Rechtsgeschichte KeJǨ, KREUZ, Prag.

2 LedVINKA, PEŠEK, Praha 277-287.

${ }^{3}$ KREUZ, Arbitrážní (ubrmanské) výroky.
}

schichten des spätmittelalterlichen Prags. Die Teilhabenden der vor dem Stadtgericht verhandelten Streite waren aber nicht nur die Einwohner Prags, sondern auch Bürger und Einwohner weiterer böhmischer Städte, Angehörige des Adels, Juden, Ausländer und weitere physische und rechtliche Personen. In den letzten Jahren wurden aus Jagiellonischer Zeit stammende Prager Bücher der Befunde unter anderem als eine Quelle zum Studium von einzelnen Formen und Typen der vor Gericht verhandelten Beleidigungen und Delikte gegen die Ehre in der ersten Hälfte des 16. Jahrhunderts ${ }^{4}$ sowie zur Erkenntnis der zwischen den Pragern und Nürnbergern bestehenden Beziehungen und vorkommenden Streitigkeiten in der Zeit der Jagiellonen herangezogen. ${ }^{5}$ Sie ermöglichten auch einen Einblick in die magischen Vorstellungen und Praktiken der Einwohner Prags im ersten Viertel des 16. Jahrhunderts. ${ }^{6}$

Die gefällten Urteile und Befunde des Stadtgerichts der Prager Altstadt (bzw. des Gerichts der in den Jahren 1518-1528 vereinigten Prager Städte) sind in den sog. Büchern der Befunde (libri sententiarum) niedergeschrieben. ${ }^{7}$ Die kontinuierliche Reihe der betreffenden altstädtischen Bücher beginnt mit dem Jahre 1479 und endet 1611, wobei sie auch ein der gerichtlichen Tätigkeit der vereinigten Prager Städte entsprungenes analoges Buch enthält. ${ }^{8}$

Die älteren Befunde des Prager Altstädter Gerichts sind in einigen vermischten Gedenk- und Eintragungsbüchern aus der zweiten Hälfte des 14. Jahrhunderts und aus dem 15. Jahrhundert verstreut. ${ }^{9}$ Aus diesen Büchern ist für die in Frage kommende Zeit nur das Buch der Ein-

\footnotetext{
${ }^{4}$ KREUZ, Urážka na cti 139-149.

${ }^{5}$ KREUZ, Spory Norimberčanů.

${ }^{6}$ KREUZ, Kouzelnictví.

${ }^{7} \mathrm{Zu}$ den Prager Gerichtsbüchern siehe detaillierter KREUZ, Odraz rozhodovací činnosti.

${ }^{8}$ ČAREK u.a., Městské a jiné úřední knihy 52.

${ }^{9}$ Ebd.
} 
schreibungen aus den Jahren 1452-1479 von Bedeutung, das insgesamt 23 Gerichtsbefunde und Urteile aus den Jahren 1475-1478 enthält. ${ }^{10}$ An diese Einschreibungen knüpft fast unmittelbar das älteste Prager altstädtische Buch der Befunde an, ${ }^{11}$ das die Zeitspanne der Jahre 14791531 umfasst. ${ }^{12}$ Ein mit zeitlichem Abstand folgendes Buch dieses Typs stellt das Buch der Befunde der vereinigten Prager Städte ${ }^{13}$ für die Jahre $1518^{14}$ bis $1527^{15}$ dar.

In den obengenannten die Befunde enthaltenden Prager Büchern (d.h. Hs. 1128, 1129 und 2141) habe ich für die Periode seit dem Jahre 1471 (resp. 1475) bis zum Jahre 1526 insgesamt 1004 Gerichtsstreite verzeichnet. Ich ließ dabei die Gruppe der 22 Fälle aus den Jahren 15001524, die stricto sensu nicht als Gerichtsstreite einzustufen waren, außer Betracht. Diese Fälle wurden nicht durch gerichtliches Urteil (gerichtlichen Befund) abgeschlossen, sondern durch einen Arbitrage-, Schieds- oder Schlichtungsspruch, dem sog. Übermannspruch. Darüber hinaus wurde in diesen Fällen das Verfahren in der Regel nicht mit einer Privatklage eröffnet. Größtenteils lag die Entscheidung in diesen Fällen nicht nur beim Stadtgericht, sondern bei einem breiteren aus Arbitern oder Schieds-, bzw. Friedensrichtern bestehenden Kollegium. ${ }^{16}$ Aus den genannten 1004 Streiten wird ein etwas größerer Teil von 533 vor dem Altstädtischen Gericht zur Verhandlung ausgetragenen Streitfällen gebildet, das heißt von Fällen aus der von der Thronbesteigung der Jagiellonen an verflossenen Zeit bis zur Vereinigung der Prager Städte. Die restlichen 471 Streite gehören in die Zeit nach Vereinigung der Prager Städte, sie ent-

\footnotetext{
${ }^{10}$ AMP, SR, Hs. 2141, fol. 171 v-186v .

11 AMP, SR, Hs. 1128.

12 ČAREK u.a., Městské a jiné úřední knihy 52.

${ }^{13}$ AMP, SR, Hs. 1129.

${ }^{14}$ Ebd., fol. 412 ${ }^{\mathrm{r}}-412^{\mathrm{v}}$.

${ }^{15}$ Ebd, fol. 36v.

16 Siehe oben FN 3.
}

stammen namentlich der Periode vom Oktober 1518 bis zum Ende des Jahres 1526. Diese Streite wurden durch das Stadtgericht der vereinigten Prager Städte verhandelt.

In der bis 1518 stattfindenden Tätigkeit des Stadtgerichts von Prag Altstadt und in dem seit 1518 einsetzendem Wirken des Gerichts der vereinigten Prager Städte lassen sich zwar manche Gemeinsamkeiten, aber auch zahlreiche auffallende Unterschiede sowohl rein quantitativen als auch proportionellen Charakters feststellen. Das Wirken des zweiten genannten Gerichts bezog sich auf eine städtische Agglomeration, die zweimal so viele Einwohner zählte, was eine eindeutige Intensivierung der Aktivitäten des Stadtgerichts zur Folge hatte. Während z. B. das Prager altstädtische Gericht in den Jahren 1475-1499 insgesamt 109 Streitfälle verhandelte und entschied, d.h. durchschnittlich nur 4 Streitfälle jährlich und in den Jahren 1510-1517 241 Streitfälle, d.h. annähernd $30 \mathrm{im} \mathrm{Jahr,} \mathrm{ent-}$ schied das Gericht der vereinigten Prager Städte in den Jahren 1519-1526 in 459 Streitfällen, d. h. durchschnittlich in 57 Verfahren jährlich.

Das Stadtgericht der Prager Altstadt (und in den Jahren 1518-1528 das Gericht der vereinigten Prager Städte) übte bis zum Jahre 1547 die Funktion des obersten Gerichtsstuhls für die Städte des altstädtischen (süddeutschen) Rechtskreises im Königreich Böhmen aus. Aus dieser Tätigkeit erhielten sich aber nur zwei Bücher der Befunde aus den Jahren 1510-1544, resp. 1510-1546.17

Mehr als ein Viertel der untersuchten Fälle bezog sich auf strafrechtliche Angelegenheiten,

${ }^{17}$ Es geht um ein Buch der Rechtsfindungen und belehrungen in Český Brod, Kouřim, Hradec Králové [Königgrätz] und Jaroměř von den Jahren 1510-1546 (AMP, SR, Hs. 93) und ein Buch der Rechtsfindungen und -belehrungen in Žatec (dt. Saaz) von den Jahren 1510-1544 (AMP, SR, Hs. 92). - AMP, SR, Hs. 93 gab J. Teige heraus - TeIge (Hg.), Zprávy o statcích, Bd. 26, 48-103, 244-288, 325-362, 433-552. AMP, SR, Hs 92 gab heraus TeIGE, Zprávy o statcích, Bd. 28, 397-637. Detailliert KREUZ, Odraz. 
den Rest stellten vorwiegend (im breitesten Sinne des Wortes) zivilrechtliche Streitigkeiten dar. Von den erwähnten 1004 Streitfällen stellten insgesamt $257(25,6 \%)$ strafrechtliche Streitfälle dar. Erwähnenswert ist der markant niedrigere Anteil von strafrechtlichen Streitigkeiten unter den vor das Stadtgericht der Prager Altstadt bis 1518 gelangten Fällen (97 Streitigkeiten von 533, d. h. bloße 18,2\%). Demgegenüber machte der Anteil der nach dem Jahre 1518 durch das Stadtgericht der vereinigten Prager Städte verhandelten strafrechtlichen Fälle 33,3 \% (157 von 471) aus. Einen annähernd gleichen Anteil (30$35 \%$ ) von strafrechtlichen Fällen verzeichnet man bezüglich der im Akkusationsverfahren vor dem Stadtgericht der Prager Altstadt verhandelten Fälle seit den 30er bis mindestens $\mathrm{zu}$ den 70er Jahren des 16. Jahrhunderts. ${ }^{18}$

Die vor dem Stadtgericht im Akkusationsverfahren verhandelten Fälle vermögen nur ein Bild eines Teils der Straftätigkeit und strafrechtlicher Gerichtsagende im jagiellonischen Prag zu bieten. Aus keiner der Prager Städte sind uns aus dem Spätmittelalter oder der Frühen Neuzeit die sogenannten Schwarzen Bücher (auch Pech- oder Blutbücher) überliefert, das sind aufgenommene Protokolle während des Vollzugs der Halsgerichtsbarkeit, die unter anderem Folteraussagen enthalten. In den Schwarzen Büchern sind dabei die im Inquisitionsverfahren verhandelten Fälle erfasst, in der Regel schwerwiegendere Kriminalität, insbesondere Eigentums- und gewaltsamen Charakters. Spezialisierte Schwarze Bücher wurden dabei in Prag schon spätestens seit der Zeit der Jagiellonen geführt - ihrer ersten Erwähnung kann man beim Gericht der vereinigten Prager Städte im Jahre 1520 begegnen. ${ }^{19}$

Während die strafrechtliche Agenda der Stadtgerichte, insbesondere die im Inquisitionsverfahren in den Böhmischen Ländern im Spätmit-

\footnotetext{
18 Vgl. dazu KREUZ, Ženy.

${ }^{19}$ KReuZ, Odraz 345-347.
}

telalter und in der Frühen Neuzeit zur Verhandlung gekommenen Fälle, in der tschechischen Historiographie beginnend mit den 1970er Jahren Gegenstand intensiver Forschung wurde, ${ }^{20}$ war die zivilrechtliche Agenda der betreffenden Gerichte bisher nur im kleinen Umfang ein Objekt eines systematischen Forschungsinteresses.

Wie schon gesagt, können die vor dem Stadtgericht im Akkusationsverfahren verhandelten strafrechtlichen Fälle nur einen Teil der Straftätigkeit im jagiellonischen Prag vermitteln. Es handelt sich vor allem um die Delikte gegen die Ehre, verschiedene Ausschreitungen und kleinere Eigentumsdelikte. Dabei lässt es sich doch nicht eindeutig konstatieren, dass es sich in allen diesen Fällen ausschließlich um weniger schwerwiegende Kriminalität gehandelt hat.

In den obengenannten 257 Fällen verzeichnete ich insgesamt 385 Straftaten. Ich gliederte sie nach dem Klassifikationsschema, das standardmäßig in der tschechischen Forschung über die Geschichte des Strafrechts und der Kriminalität schon seit Ende der 1970er Jahre in Geltung ist, ${ }^{21}$ in insgesamt 5 Gruppen, und zwar: 1. Delikte gegen den Staat, die öffentliche Macht und ihre Repräsentanten; 2. Delikte gegen die Rechtsordnung, die Sicherheit und öffentliche Ordnung; 3. Delikte gegen das Eigentum; 4. Delikte gegen das Leben und die Gesundheit; 5. Delikte gegen persönliche Rechte mit den Untergruppen: 5a) Delikte gegen die persönliche Freiheit; 5b) Delikte gegen die Ehre; 5c) Delikte gegen die Ehe und sexuelle Delikte.

Am niedrigsten war der Anteil der Klagen wegen Delikte gegen den Staat, die öffentliche

\footnotetext{
${ }^{20}$ Für eine Übersicht dieser Forschung siehe vor allem PÁNEK, Městské hrdelní soudnictví; PÁNEK, Die Halsgerichtsbarkeit; FRANCEK, ŠIMEK, Hrdelní soudnictví 134-179; ProCHÁZKOVÁ, Současný stav; KREUZ, Dějiny pozdně středověkého a raně novověkého městského soudnictví.

${ }^{21}$ MALÝ, Trestní právo.
} 
Macht und ihre Repräsentanten. ${ }^{22}$ Es wurden deren nur 20 erfasst (das sind 5,2\%). Von einzelnen Delikten ist am häufigsten eine Beleidigung des Stadtgerichts, eines Mitglieds des Stadtrates oder eines städtischen Beamten vorgekommen (9 Fälle). Man begegnet auch häufiger dem Missbrauch der Amts- oder Richtersvollmacht (4 Fälle) und der Blasphemie/ Gotteslästerung (3). Aus Gründen, die im Falle des jagiellonischen Prag eine ausführlichere Darstellung verdienten, begegnen wir hier überhaupt nicht dem Delikt der Ketzerei und nur marginal dem Magiedelikt, und zwar in Form der Zauberei (Schadenzauber), nicht in Form der (diabolischen) Hexerei (des kumulativen Magiedelikts). ${ }^{23}$

Als zahlreicher erwiesen sich in den eingereichten Klagen Delikte gegen die Rechtsordnung, Sicherheit und öffentliche Ordnung, ${ }^{24}$ von denen insgesamt 53 (d.h. 13,8\%) zutage treten. Wenigstens einige in diese Gruppe von Delikten fallende Fälle stellten Gewalthandlungen (insbesondere Ausschreitung und Gewalt) dar, die eben typisch waren für das Stadtmilieu des späten Mittelalters und der Frühen Neuzeit. Die am häufigsten festgestellten Straftaten innerhalb der obengenannten Gruppe von Delikten war die schon erwähnte Gewalt und gefährliche Drohung; beide Delikte kamen in 11 Fällen vor. Als weitere, relativ häufiger vorkommende Delikte innerhalb der betreffenden Gruppe sind zu nennen die Ausschreitung (9 Fälle), Nichtbefolgung eines Gerichtsurteils (7) und Unterstützung eines Verbrechers (7). ${ }^{25}$ In vier Fällen wurde Anklage wegen pych (Frevel) erhoben. Das schon erwähnte Delikt der öffentlichen Gewalt (moc) taucht in zwei Fällen auf. In einem Falle galt die Klage einem weiteren, typisch landesrechtlichen Delikt, und zwar die Fehdeerklärung (od-

\footnotetext{
${ }^{22}$ Vgl. ebd. 39-79.

${ }^{23}$ KREUZ, Kouzelnictví 131, 136-143.

${ }^{24} \mathrm{Vgl}$. MALÝ, Trestní právo 81-117.

${ }^{25}$ Ebd. 95-100.
}

pověd'). ${ }^{26}$ Odpověd' weist mehrere gemeinsame Züge mit der mittelalterlichen Fehde ${ }^{27}$ auf, die man in manchen Gebieten des Reiches antrifft. ${ }^{28}$

Die Gruppe der Delikte gegen das Eigentum (Eigentumsdelikte) ${ }^{29}$ kommt in den Klagen in relativ unerheblicher Zahl vor. Es fallen darunter nur 54 (d.h. 14,0\%) der zum Gegenstand einer Klage gewordenen Delikte. Auf der anderen Seite weist sie eine ziemlich differenzierte Struktur auf, weil sich in ihr insgesamt 9 Arten von Delikten unterscheiden lassen. Für die im Akkusationsverfahren verhandelten Straffälle sind gegenüber den im Inquisitionsverfahren zur Verhandlung gelangenden Fälle gerade die relativ geringe Häufigkeit der Eigentumsdelikte einerseits und ihre erhebliche Differenzierheit nach ihren Arten charakteristisch. Unter den festgestellten Delikten entfällt der größte, aber nicht mehrheitliche Anteil auf das Delikt des Diebstahls (21 Fälle). In relativ größerer Zahl (8 Fälle) verzeichnen wir auch das Delikt der Hehlerei und des Verkaufs der gestohlenen Sachen. Der Raub kommt in 5 Fällen auf und die unberechtigte Aneignung einer fremden Mobilie (beweglichen Sache oder fremden Viehs) in 7 Fällen. Das schwerwiegende Delikt der Brandstiftung figuriert in drei Fällen. Der Betrug, einschließlich der Fälschung der feilgebotenen Ware, kommt in 4 Fällen vor.

Bedeutend zahlreicher waren die Klagen wegen Delikte gegen das Leben und die Gesundheit. ${ }^{30}$ Es wurden insgesamt 122 solcher Delikte registriert $(31,7 \%)$. Die Klagen bezogen sich vor allem auf Delikte, zu denen es im Laufe von mit Gewalt begleiteten Konflikten und Zusammenstößen in den Straßen oder öffentlichen Räumen der Stadt kommen sollte. Diese Tatsache bestätigt die Vorstellung der neueren Forschung über

\footnotetext{
${ }^{26}$ Vgl. AMP, SR, Hs. 1129, fol. 431 v $-432^{\mathrm{v}}\left(432^{\mathrm{v}}-433^{\mathrm{v}}\right)$.

${ }^{27}$ KAUFMANN, Fehde.

${ }^{28}$ MALÝ, Trestní právo 88-91.

${ }^{29}$ Ebd. 119-165.

${ }^{30}$ Ebd. 167-200.
} 
die Stadt des Spätmittelalters als Konfliktgemeinschaft, die sich durch intensive und vielfältige soziale Kommunikation auszeichnet. ${ }^{31}$ In 36 Fällen wurde Mord zum Gegenstand der Klage und in 18 Fällen ein Mordversuch. Am häufigsten begegnet man aber den Klagen wegen Verletzung/Gesundheitsschädigung (38 Fälle). Damit waren Klagen wegen Prügelung eng verknüpft (26). Nur in ganz seltenen Fällen kann man den Gegenstand der Klage als Gesundheitsschädigung mit Todesfolge (2) charakterisieren. Dies zeigt, dass die meisten Fälle der Tötung im gewaltsamen Konflikt bei dem Stadtgericht als Mord angeklagt wurden, und zwar meist unabhängig vom tatsächlichen Verlauf des Konflikts. In die oben genannte Gruppe fallen auch ein Fall von Vergiftung und eine Klage wegen ungerechtfertigter Tortur. ${ }^{32}$

Die größte Gruppe der angeklagten Delikte bilden die Delikte gegen persönliche Rechte, ${ }^{33}$ deren insgesamt 136 festgestellt wurden (35,3\%). Dieser Umstand ist vor allem darauf zurückzuführen, dass hier die Untergruppe der Delikte gegen die $E^{2}{ }^{34}$ ins Gewicht fällt, von denen insgesamt 121 vermerkt wurden (31,4\%), während die wegen Delikte gegen die persönliche Freiheit ${ }^{35}$ (7 Fälle, d.h. 1,8 \%) und wegen Delikte gegen die Ehe und sexueller Delikte ${ }^{36}$ (8 Fälle, d.h. 2,1\%) eingereichten Klagen in bedeutend kleinerer Zahl auftreten.

Die innerhalb der Untergruppe der Delikte gegen die persönliche Freiheit eingereichten Klagen bezogen sich konkret auf die ungerechtfertigte Einkerkerung, gewaltsame Erzwingung einer Verpflichtung und die Beschränkung der persönlichen Freiheit.

\footnotetext{
${ }^{31}$ Dazu z.B. SchUSTER, Eine Stadt vor Gericht 61-72, 86-104.

${ }^{32}$ AMP, SR, Hs. 1128, fol. $11^{\mathrm{r}}-11^{\mathrm{v}}\left(2^{\mathrm{r}}-2^{\mathrm{v}}\right)$.

${ }^{33}$ MALÝ, Trestní právo 201-261.

${ }^{34}$ Ebd. 205-231; BOROVSKÝ, u.a., Spory o čest.

${ }^{35}$ MALÝ, Trestní právo 201-204.

${ }^{36}$ Ebd. 231-246. Überwiegend für die Stadtgerichte KREUZ, Zur schweren Sexualdelinquenz.
}

Das am häufigsten angeklagte Delikt gegen die Ehre und zugleich das am häufigsten von den oben genannten Prager Gerichten in der jagiellonischen Zeit verhandelte Delikt war die Ehrenbeleidigung (95 Fälle). Mit großem Abstand folgten Klagen wegen sogenannter Schmähung (24 Fälle), die (kurz und ein bisschen vereinfacht gesagt) eine Beleidigung von geringerer Intensität darstellte, die keine direkte Anzweiflung der Ehre des Klägers war. ${ }^{37}$

In der Untergruppe Ehe- und Sexualdelikte treffen wir zwei Klagen wegen sogenannter Entlockung (ulouzení) einer verheirateten Frau oder eines verheirateten Mannes, ${ }^{38}$ die auch die Form der Entführung der Ehefrau annehmen konnte, und auch zwei Klagen wegen Konkubinat. In zwei Fällen wurde eine Klage wegen schlechter Behandlung der Ehefrau vorgebracht, die konkret die Gestalt der Misshandlung der eigenen Frau gewann, beziehungsweise Verjagung der Ehefrau aus dem Hause. Die Unzucht wurde zum Klagegegenstand nur in einem Falle, ebenso die Vergewaltigung.

Das am häufigsten unter den vor dem Stadtgericht von Prager Altstadt beziehungsweise dem Stadtgericht der vereinigten Prager Städte in der jagiellonischen Zeit im Akkusationsverfahren verhandelte strafrechtliche Delikte stellte das Delikt der Ehrenbeleidigung dar (95). Mit groBem Abstand folgen drei Delikte gegen das Leben und die Gesundheit, und zwar Verletzung (38), Mord (36) und Verprügelung (26). Die Reihe der fünf am häufigsten einen Gegenstand der Klage bildenden Delikte schließt die Schmähung ab (24). Von den restlichen Deliktsgruppen ist am häufigsten als Klagsgegenstand der Diebstahl zu nennen, und zwar in 21 Fällen. Von weiteren Delikten erreichten die zweistellige Vertretung nur Mordversuch (18), Androhung

\footnotetext{
${ }^{37}$ Vgl. auch KREUZ, Urážka na cti 141-143.

${ }^{38} \mathrm{Zu}$ diesem Delikt MALÝ, Trestní právo 237-240; detaillierter KREUZ, Zur schweren Sexualdelinquenz 159-161.
} 
(11) und Gewalt (11). Von den 40 Arten von angeklagten Straftaten, die bei den untersuchten Gerichten verzeichnet worden sind, entfielen also auf die relativ häufiger vorkommenden nur neun von ihnen. Von diesen wurden annähernd vier Fünftel der verhandelten Delikte gebildet.

Aufgrund der vorliegenden kurzen Übersicht wird deutlich, dass unter den aufgrund einer Privatklage im Akkusationsverfahren vor den untersuchten im jagiellonischen Prag tätigen Gerichten die Delikte gegen die Ehre und die Delikte gegen das Leben und die Gesundheit mehrheitlich in Erscheinung traten. Auf sie entfielen die meisten verhandelten Fälle, fast zwei Drittel aller vor die obengenannten Gerichte gelangenden Klagen. Die Delikte gegen das Leben und die Gesundheit kamen öfter im $\mathrm{Zu}$ sammenhang mit einigen Delikten gegen die öffentliche Ordnung vor. Einen relativ häufiger vorkommenden Gegenstand stellten auch einige Straftaten aus dem Gebiet der Eigentumsdelikte dar, die schwieriger zu beweisen sein konnten. Schwer beweisbar waren manchmal auch die Klagen wegen Delikte gegen das Leben (insbesondere Mord).

Aus dem Gesichtspunkt der Entwicklung des Stadtrechts betrachtet, lässt sich während der jagiellonischen Epoche eine deutliche Vervollkommnung der Entscheidungstätigkeit und der Befundpraxis des Altstädter Stadtgerichts feststellen. Diese Vervollkommnung projizierte sich in die schon in der Regel formell der gewünschten Qualität entsprechende und umfangreiche Begründungen der Urteile und Befunde des Gerichts der vereinigten Prager Städte nach 1518 hinein. Während das Prager Altstädter Recht noch an der Schwelle der Zeit der Jagiellonnen von seiner Entwicklung her ein Recht sui generis, ein in erheblichem Maße autochtones Rechtssystem darstellte, das nur fallweise und unsystematisch die Normen des sächsischen oder Magdeburger Rechts, bzw. süddeutschen Rechts übernahm, standen die ersten Dezennien der jagiellonischen Epoche der Prager Altstadt im Zeichen der breiten und intensiven Rezeption des bei weitem vollkommeneren Brünner Stadtrechts und Brünner-Iglauer Rechts und durch ihre Vermittlung auch des römischen Rechts. Diese Rezeption, bzw. Annahme, die eine der bedeutsamen Voraussetzungen späterer Kodifikation des böhmischen Stadtrechts (1579) bildete, war in Prag an der Neige der jagiellonischen Epoche fast vollendet sowohl in theoretischer Ebene als auch in der Sphäre der Rechtspraxis. ${ }^{39}$

\section{Korrespondenz:}

Doz. DDr. Petr KREUZ

Archiv hlavního města Prahy

Archivní 6

CZ - 14900 Praha 4

Česká republika

Petr.Kreuz@praha.eu

ORCID-Nr. 0000-0001-7484-4773

\section{Abkürzungen:}

\section{AMP Archiv hlavního města Prahy [Archiv der Hauptstadt Prag] \\ SR Sbírka rukopisů [Handschriftensammlung]}

Siehe auch das allgemeine Abkürzungsverzeichnis: [http://www.rechtsgeschichte.at/media/abk.pdf]

\footnotetext{
${ }^{39}$ Neu zu diesem Thema KreuZ, Pavel Kristián von Koldín Stadtgesetzbuch.
} 


\section{Literatur:}

Tomáš BOROVSKÝ, Dalibor JANIŠ, Michaela MALANÍKOVÁ u.a., Spory o čest ve středověku a raném novověku (Brno 2010).

Jiří ČAREK u.a., Městské a jiné úřední knihy Archivu hlavního města Prahy (Přehled) (Praha 1956).

Jindřich FRANCEK, Tomáš ŠIMEK (Hgg.), Hrdelní soudnictví českých zemí. Soupis pramenů a literatury (Zámrsk, Pardubice 1995).

E. KAUfMANN, Fehde, in: Adalbert ERLER, Ekkehard KAUFMANN (Hgg.), Handwörterbuch zur deutschen Rechtsgeschichte, Bd. 1 (Berlin 19711) 1083-1093.

Jiří KeJর̌, Petr KReuZ, Prag, in: Albrecht CORDES u.a. (Hgg.), Handwörterbuch zur deutschen Rechtsgeschichte, Bd. 3 (Berlin 22013) 711-718.

Petr KREUZ, Ženy $\mathrm{v}$ trestněprávních případech před městským soudem Starého Města pražského v letech 1548-1560, in: Documenta Pragensia 13 (1996) 101-134.

DERS., Urážka na cti v praxi komorního soudu Království českého a městského soudu Starého Města pražského v první polovině 16. století in: BOROVSKÝ, JANIŠ, MALANÍKOVÁ u.a., Spory o čest 127-150.

DERS., Spory Norimberčanů a Pražanů před městským soudem Starého Města pražského v době jagellonské, in: Olga FejTOvá, Václav LEDVINKA, Jiří PEŠEK (Hgg.), Ztracená blízkost. Praha - Norimberk v proměnách století (= Documenta Pragensia 29, Praha 2010) 147-175.

DERS., Kouzelnictví a jeho pronásledování v jagellonské Praze. Příspěvek $\mathrm{k}$ počátkům kouzelnických a čarodějnických procesů v Čechách, in: Pražský sborník historický 40 (2012) 131-164.

DERS., Dějiny pozdně středověkého a raně novověkého městského soudnictví v českých zemích $\mathrm{v}$ české historiografii posledních dvaceti let, in: Olga FejTOVÁ, Michaela HRUBÁ, Václav LEDVINKA, Jiří PEŠEK, Ludmila SuliTKOVÁ (Hgg.), Města ve středověku a raném novověku jako badatelské téma posledních dvou desetiletí (=Documenta Pragensia 32/2, Praha 2013) 179-199.

DERS., Zur schweren Sexualdelinquenz vor den böhmischen Stadtgerichten im 16. und beginnenden 17. Jahrhundert, in: Gerhard AMMERER, Gerhard FRITZ, Jaromír TAUCHEN (Hgg.), Sexualität vor Gericht. Deviante geschlechtliche Praktiken und deren Verfolgung vom 14. bis zum 19. Jahrhundert
(= Beiträge zur Rechtsgeschichte Österreichs 9/1, Wien 2019) 158-172.

DERS., Odraz rozhodovací činnosti městského soudu Starého Města Pražského a městského soudu spojených pražských měst $\mathrm{v}$ pražských městských knihách z doby jagellonské, in: Jan HRDINA, Kateřina JíšovÁ (Hgg.), Úřední písemná kultura $\mathrm{v}$ českých a moravských městech ve středověku a raném novověku (Praha 2019) 339-348.

DERS., Pavel Kristián von Koldín Stadtgesetzbuch von 1579 und mittelalterliche Stadtrechte in den Böhmischen Ländern, in: Andreas DeuTsCH (Hg.), Stadtrechte und Stadtrechtsreformationen. Tagungsband vom Symposium in Heidelberg von 3. 4. bis 5. 4. 2019 (im Druck).

DERS., Arbitrážní (ubrmanské) výroky městského soudu Starého Města pražského a soudu spojených pražských měst $\mathrm{z}$ doby jagellonské. Př́spěvek k poznání mediace a urovnávání sporů v pozdně středověké Praze, in: Pražský sborník historický 47 (2020, im Druck).

Václav LedvinKA, Jiří Pešek, Praha (Praha 2000).

Karel MALÝ, Trestní právo v Čechách v 15. a 16. století (Praha ${ }^{21989) . ~}$

Jaroslav PÁNEK, Městské hrdelní soudnictví v pozdně feudálních Čechách (Výsledky, problémy a perspektivy studia), in: Československý časopis historický 32 (1984) 693-728.

DERS., Die Halsgerichtsbarkeit der böhmischen Städte und Märkte vom 16. bis 18. Jahrhundert, in: MIÖG 96 (1988) 95-131.

Eva ProchÁzKovÁ, Současný stav výzkumu dějin kriminality a soudní praxe $\mathrm{v}$ éře decentralizovaného soudnictví, in: Jiří PEŠEK (Hg.), VIII. sjezd českých historiků. Hradec Králové 10.-12. záŕí 1999 (Praha 2000) 178-189.

Peter SCHUSTER, Eine Stadt vor Gericht. Recht und Alltag im mittelalterlichen Konstanz (Paderborn u.a. 2000).

Josef TeIge (Hg.), Zprávy o statcích a přech venkovských z archivu města Prahy, A-K, in: Archiv český čili staré písemné památky české i moravské sebrané z archivů domácích i cizích, Bd. 26 (Praha 1909).

Ders. (Hg.), Zprávy o statcích a přech venkovských z archivu města Prahy, L-Z, in: Archiv český čili staré písemné památky české i moravské sebrané z archivů domácích i cizích, Bd. 28 (Praha 1912). 